\title{
Hypoxemia during surgery: learning from history, science, and current practice
}

\author{
Gregory M. T. Hare, MD, PhD • \\ Brian P. Kavanagh, MD
}

Published online: 27 July 2010

(C) Canadian Anesthesiologists' Society 2010

Prevention of hypoxemia remains one of the primary goals related to the safe practice of anesthesia. Development of the pulse oximeter has allowed for the reliable detection and prompt treatment of hypoxemia. As reviewed by Drs. Ehrenfeld et al. in this issue of the Journal, routine intraoperative application of pulse oximetry coupled with parallel increases in patient safety has made a significant impact on the practice of anesthesia. ${ }^{1}$ Careful conduct of anesthesia based on real-time knowledge of blood oxygenation $\left(\mathrm{S}_{\mathrm{p}} \mathrm{O}_{2}\right)$ stands in stark contrast with anesthesia practice patterns of previous eras in which short-term maintenance of anesthesia with high concentrations of nitrous oxide was regarded as effective and safe in healthy pre-oxygenated patients. ${ }^{2}$ With the invention and use of the oxygen electrode, ${ }^{3}$ it gradually became apparent that profound decreases in arterial oxygen tension occurred with the former method of anesthetic induction. Nonetheless, several clinical studies were continued wherein high

G. M. T. Hare, MD, PhD

Department of Anesthesia, Keenan Research Centre in the Li Ka Shing Knowledge Institute and the Cara Phelan Centre for Trauma Research, St. Michael's Hospital, Toronto, ON, Canada

B. P. Kavanagh, MD

Departments of Critical Care Medicine and Anesthesia,

The Hospital for Sick Children, Toronto, ON, Canada

G. M. T. Hare, MD, PhD - B. P. Kavanagh, MD

Department of Physiology, University of Toronto,

Toronto, ON, Canada

G. M. T. Hare, MD, PhD ( $ه)$

Department of Anesthesia and Physiology, University

of Toronto, St. Michael's Hospital, 30 Bond Street,

Toronto, ON M5B 1W8, Canada

e-mail: hareg@smh.toronto.on.ca concentrations of nitrous oxide with reciprocally low concentrations of inspired oxygen were explored. This practice led to the frequent occurrence of clinically significant hypoxemia., ${ }^{2,5}$

In their classic article published a half century ago, Heller et al. reported the following with respect to induction of anesthesia: "It is preferable to start with an 80/20 mixture of $\mathrm{N}_{2} \mathrm{O}$ and $\mathrm{O}_{2}$ rather than to subject the anxious wakeful patient to a preliminary period of $(100 \%)$ oxygen breathing." 5 Although such approaches were developed at a time of limited availability of anesthetic agents, this practice - thankfully - differs dramatically from our current approach. These days, we often advocate the avoidance of nitrous oxide, ${ }^{6,7}$ and pre-oxygenation is advocated routinely as one means to prevent hypoxemia. The large database of the current study helps us to appreciate further the frequency and risks of perioperative hypoxia, moving us forward from the days of "black gas anesthesia".

In their report in this issue of the Journal, ${ }^{1}$ Ehrenfeld et al. present the incidence of hypoxemia during surgery using data they derived from a sizeable series of electronic records from two large medical centres. The authors report that hypoxemia $\left(\mathrm{SpO}_{2}<90 \%\right)$ is infrequent $(6.8 \%)$ - but perhaps too common for an entity that anesthesiologists always try to avoid. Indeed, severe hypoxemia $\left(\mathrm{SpO}_{2}<\right.$ $85 \%$ ) occurred in $3.5 \%$ of patients. They also reported that transient hypoxemia occurred during all phases of anesthesia, especially during induction and emergence and particularly in patients who were American Society of Anesthesiologists (ASA) physical status III or IV. Aside from recognizing that we have not "conquered" intraoperative hypoxemia, what other important lessons can be learned from these data?

First, we should reiterate the most obvious positive news. Acceptable levels of $\mathrm{SpO}_{2}(>90 \%)$ were maintained 
in the overwhelming majority of patients for the majority of observations, i.e., $99.63 \%$ of 16 million recorded values. Second, the majority (99\% of events) of transient hypoxemic episodes were relatively brief, i.e., less than five minutes. Together, these simple but comprehensive descriptive data convey that the process of monitoring, observation, diagnosis, and management of hypoxemia is generally successful. Although the study was not designed to link these events with outcomes, empirically, monitoring and preventing prolonged hypoxemia would be universally desirable. This is consistent with the dramatic reduction in hypoxemia-related mortality previously described.

Is the timing of intraoperative hypoxemia important? Although hypoxemia occurred throughout anesthesia, it happened most often during induction and emergence, supporting the clinical view that these periods of anesthetic state transitioning represent periods of higher risk. Indeed, it is at these times that anesthesiologists are characteristically most vigilant. Difficulties may arise during these "vulnerable" periods, such as unanticipated airway difficulties, problems maintaining ventilation post-induction, or laryngospasm upon emergence. However, there may also be aspects of transitional physiology and pharmacology that could provide additional and perhaps more subtle explanations for some of the episodes of hypoxemia. For example, rapid exposure to inhalation anesthetics may impair optimal matching of ventilation with perfusion due to inhibition of hypoxic pulmonary vasoconstriction. ${ }^{8}$ A recent review of rapid sequence induction in children demonstrated a significant incidence of moderate and severe hypoxemia despite optimal pre-oxygenation. ${ }^{9}$ Broader implementation of a standard methodology to optimize oxygenation after tracheal intubation, including alveolar recruitment maneuvers, ${ }^{10}$ could be adequate to limit the degree of post-induction hypoxemia.

Also, subsequent events can lead to hypoxemia, such as the development of atelectasis, ${ }^{11}$ loss of spontaneous ventilation, and emergent issues, such as laryngospasm, residual paralysis, and hypoventilation from sedatives or opioids. Prevention of these events can be achieved utilizing established methods (e.g., recruitment maneuvers) ${ }^{10}$ and may be further improved by developing novel approaches (e.g., negative pressure ventilation). ${ }^{12}$ The frequent occurrence of multifactorial threats to maintain optimal oxygenation supports our current anesthesia practice of oxygen supplementation and continuous $\mathrm{O}_{2}$ monitoring. With the desire to eliminate episodes of perioperative hypoxemia, the current report prompts the following questions: Do we need to do better? If so, by what maneuvers and against what standards can improvement be achieved?

In a recent observational study, Blum et al. assessed the anesthesiologists' response to episodes of mild, moderate, and severe intraoperative hypoxemia. ${ }^{13}$ They identified that anesthesiologists attempted to correct episodes of hypoxemia by increasing the fraction of inspired oxygen $\left(\mathrm{F}_{1} \mathrm{O}_{2}\right)$, peak inspiratory pressure, and ventilation. While appropriate as "first response" maneuvers to correct insufficient $\mathrm{F}_{\mathrm{I}} \mathrm{O}_{2}$ or inadequate ventilation, these approaches do not broadly address all possible causes of hypoxemia. Thus, a clearer understanding of the mechanisms that contribute to ventilation-perfusion mismatch and shunt would optimize gas exchange. In this context, the use of positive end-expiratory pressure (PEEP) may be beneficial.

The finding that the patient's ASA status correlated with hypoxemic events is not surprising. However, this also emphasizes that patients with significant comorbidities are likely to have an increase in perioperative events such as transient hypoxemia. Further study will be required to determine if such events lead to an increased incidence of adverse clinical outcomes.

Finally, the mechanisms by which transient hypoxemia transitions from being well-tolerated and potentially beneficial (hypoxia preconditioning) to being harmful (sleep apnea, pulmonary hypertension) must be understood more clearly. The physiology of hypoxemia adaptation has demonstrated that modification of hypoxic regulatory responses may allow for adaptation to hypoxia exposure. For example, populations that adapt to lower $\mathrm{F}_{1} \mathrm{O}_{2}$ at high altitude demonstrate: 1) impaired hypoxic pulmonary vasoconstriction; 2) optimized glycolytic metabolism; 3) increased microvascular density and blood volume; and 4) increased blood oxygen carrying capacity. ${ }^{14}$ These adaptations occur at the cellular level and support the phenomenon of hypoxia preconditioning. ${ }^{15}$ Although we may never fully understand all mechanisms involved, the hypothesis that mild controlled hypoxic preconditioning may improve patient outcomes following general anesthesia and surgery could be tested clinically. Since a reductionist scientific approach to define an effective organ protective mechanism has not been clinically successful, a black-box generalized approach to determine the efficacy of mild hypoxia preconditioning may be justified. This approach must be balanced with the known risks of intermittent hypoxia, including pulmonary hypertension and mortality.

In conclusion, Ehrenfeld et al. ${ }^{1}$ must be congratulated on their observation from an extensive clinical database that intraoperative hypoxemia remains a current and ongoing concern. Practices that reduce the risk of hypoxemia in this setting, including pre-oxygenation, increased intraoperative $\mathrm{F}_{\mathrm{I}} \mathrm{O}_{2}$ concentration, and preserving adequate ventilation are essential to prevent intraoperative hypoxemia. Finally, novel approaches must be sought to minimize the frequency of anesthesia-related hypoxemia, even as basic and clinical science research is being pursued to elucidate further the perhaps more subtle contributing mechanisms. 


\section{L'hypoxémie pendant la chirurgie: les leçons de l'histoire, de la science et des pratiques actuelles}

La prévention de l'hypoxémie demeure l'un des principaux enjeux d'une pratique sécuritaire de l'anesthésie. Grâce à la mise au point de l'oxymètre de pouls, nous pouvons désormais dépister l'hypoxémie de façon fiable et la traiter rapidement. L'application peropératoire de l'oxymétrie de pouls dans la pratique de routine, combinée à une augmentation parallèle de la sécurité des patients, a eu un impact considérable sur la pratique de l'anesthésie, comme les Drs Ehrenfeld et coll. le notent dans ce numéro du Journal. ${ }^{1}$ L'administration prudente de l'anesthésie, fondée sur une connaissance en temps réel de l'oxygénation du sang $\left(\mathrm{S}_{\mathrm{p}} \mathrm{O}_{2}\right)$, est en opposition totale avec les modèles de pratique de l'anesthésie du passé. En effet, le maintien à court terme de l'anesthésie avec des concentrations élevées de protoxyde d'azote était alors considéré comme efficace et sécuritaire chez le patient sain pré-oxygéné. ${ }^{2}$ Avec l'invention et l'utilisation de l'électrode à oxygène, ${ }^{3}$ nous nous sommes petit à petit rendus compte que cette méthode d'induction de l'anesthésie provoquait des réductions importantes de la teneur en oxygène dans le sang artériel. Plusieurs études cliniques ont cependant continué à examiner des concentrations élevées de protoxyde d'azote avec des concentrations réciproquement basses d'oxygène inhalé. Cette pratique a entraîné la survenue fréquente d'épisodes d'hypoxémie cliniquement significative. ${ }^{2,4,5}$

Dans leur article qui fait autorité publié il y a un demi-siècle, Heller et coll. notaient, concernant l'induction de l'anesthésie: "Il est préférable de commencer avec un mélange à 80/20 de $\mathrm{N}_{2} \mathrm{O}$ et d' $\mathrm{O}_{2}$ plutôt que de soumettre un patient éveillé et anxieux à une période préliminaire de respiration d'oxygène à $100 \%$. ${ }^{5}$ Bien que de telles approches aient été mises au point à un moment où la disponibilité des agents anesthésiques était limitée, cette pratique est heureusement bien loin de notre approche actuelle. De nos jours, nous recommandons souvent d'éviter le protoxyde d'azote, ${ }^{6,7}$ et la préoxygénation est régulièrement recommandée comme l'une des façons de prévenir l'hypoxémie. L'importante base de données de l'étude en question ici nous permet d'apprécier pleinement la fréquence et les risques d'une hypoxie périopératoire, nous propulsant bien loin du temps de « l'anesthésie au gaz noir ».

Dans ce numéro du Journal, le compte-rendu de Ehrenfeld et coll. présente l'incidence de l'hypoxémie pendant la chirurgie; les données sont dérivées d'une importante série de dossiers électroniques provenant de deux grands centres médicaux. Les auteurs rapportent que l'hypoxémie $\left(\mathrm{SpO}_{2}<90 \%\right)$ est peu fréquente $(6,8 \%)$, mais tout de même trop courante pour une entité que les anesthésiologistes tentent toujours d'éviter; en effet, une hypoxémie grave $\left(\mathrm{SpO}_{2}<85 \%\right)$ a été observée chez $3,5 \%$ des patients. Les auteurs rapportent aussi qu'une hypoxémie transitoire a été observée pendant toutes les phases de l'anesthésie, particulièrement pendant l'induction et le réveil, et tout particulièrement chez les patients de classe ASA III ou IV. Outre la prise de conscience que nous n'avons pas encore «dompté » l'hypoxémie peropératoire, quelles sont les leçons importantes à retirer de ces données?

En premier lieu, il convient de réitérer les bonnes nouvelles les plus évidentes. Des niveaux acceptables de $\mathrm{SpO}_{2}(>90 \%)$ ont été maintenus chez la vaste majorité des patients et pour la majorité des observations-soit 99,63\% des 16 millions de valeurs enregistrées. Deuxièmement, la majorité $(99 \%)$ des épisodes d'hypoxémie transitoire étaient relativement courts (c.-à-d. $<5$ minutes). Lorsque combinées, ces données descriptives simples mais complètes reflètent le fait que le processus de monitorage, d'observation, de diagnostic et de prise en charge de l'hypoxémie est en général réussi. Bien que l'étude n'ait pas été conçue pour lier ces événements à des devenirs, nos connaissances empiriques nous laissent à penser que le monitorage et la prévention d'une hypoxémie prolongée seraient des éléments universellement souhaitables. Ceci concorde avec la réduction spectaculaire de la mortalité liée à l'hypoxémie précédemment mentionnée.

Le moment de survenue d'une hypoxémie peropératoire est-il important? Quoique des épisodes d'hypoxémie aient été observés tout au long de l'anesthésie, ils étaient le plus fréquent pendant l'induction et le réveil, ce qui va dans le sens de l'opinion clinique que ces périodes de transition de l'état anesthésique sont des périodes à risque plus élevé. C'est en effet à ces moments-là que les anesthésiologistes sont en général les plus vigilants. Pendant ces périodes de 'vulnérabilité', des difficultés imprévues au niveau des voies aériennes peuvent survenir, ou des problèmes pour maintenir la ventilation après l'induction, ou encore un laryngospasme pendant la phase de réveil. Cependant, certains aspects de la physiologie et de la pharmacologie pendant la période de transition pourraient expliquer davantage, et de façon plus subtile peut-être, certains des épisodes d'hypoxémie. Par exemple, l'exposition rapide à des anesthésiques par inhalation pourrait gêner le pairage optimal de la ventilation à la perfusion en raison de l'inhibition de la vasoconstriction pulmonaire hypoxique. ${ }^{8}$ Une revue récente de l'induction en séquence rapide chez l'enfant a révélé une incidence significative d'hypoxémie modérée à grave et ce, malgré une préoxygénation optimale. ${ }^{9}$ L'application plus répandue 
d'une méthodologie normalisée afin d'optimiser l'oxygénation après l'intubation trachéale, comprenant notamment des manœuvres de recrutement alvéolaire, ${ }^{10}$ pourrait être utile si l'on souhaite limiter quelque peu l'hypoxémie après l'induction.

Des événements subséquents peuvent également provoquer l'hypoxémie, comme par exemple l'apparition d'atélectasie, ${ }^{11}$ la perte de respiration spontanée, et des problèmes au réveil tels que le laryngospasme, une paralysie résiduelle et l'hypoventilation dus aux sédatifs ou aux opioïdes. On peut prévenir ces événements en ayant recours à des méthodes établies (par ex., des manœuvres de recrutement), ${ }^{10}$ et la prévention peut être encore améliorée grâce à la mise au point de techniques innovantes (par ex., la ventilation en pression négative). ${ }^{12} \mathrm{La}$ survenue fréquente de menaces multifactorielles au maintien d'une oxygénation optimale appuie notre pratique actuelle de l'anesthésie, qui consiste en l'addition d'oxygène et le monitorage continu de $\mathrm{l}^{\prime} \mathrm{O}_{2}$. Avec le but d'éliminer les épisodes d'hypoxémie périopératoire, le compte-rendu présenté ici suscite la question suivante: devons-nous faire mieux et, le cas échéant, à l'aide de quelles manœuvres et par rapport à quelles normes?

Dans une étude observationnelle récente, Blum et coll. ont évalué la réaction des anesthésiologistes aux épisodes d'hypoxémie peropératoire légère, modérée et grave. ${ }^{13}$ Ils ont remarqué que les anesthésiologistes tentaient de corriger les épisodes d'hypoxémie en augmentant la fraction d'oxygène inhalé $\left(\mathrm{F}_{\mathrm{I}} \mathrm{O}_{2}\right)$, la pression inspiratoire de pointe et la ventilation. Bien qu'elles soient adaptées en tant que manœuvres de «première intervention » pour corriger une $\mathrm{F}_{\mathrm{I}} \mathrm{O}_{2}$ insuffisante ou une ventilation inadéquate, ces approches ne traitent pas largement toutes les causes possibles d'hypoxémie. Dès lors, une meilleure compréhension des mécanismes qui contribuent au décalage entre la ventilation et la perfusion et au shunt pourrait optimiser l'échange gazeux. Dans ce contexte, la pression positive télé-expiratoire (PEEP) pourrait être avantageuse.

La découverte que la classe ASA du patient est corrélée aux événements hypoxémiques n'est pas surprenante. Toutefois, cela souligne le fait que les patients présentant d'importantes comorbidités seront probablement plus susceptibles à des événements périopératoires tels que l'hypoxémie transitoire. Des recherches plus approfondies seront nécessaires pour déterminer si de tels événements entraînent une incidence accrue de devenirs cliniques défavorables.

Enfin, une meilleure compréhension des mécanismes par lesquels une hypoxémie transitoire se transforme d'un état bien toléré, voire potentiellement bénéfique (préconditionnement hypoxique) à un état nocif (apnée du sommeil, hypertension pulmonaire) est essentielle. La physiologie de l'adaptation à l'hypoxémie a démontré que la modification des réponses de régulation hypoxique pourrait permettre une adaptation à l'exposition à l'hypoxie. Par exemple, les populations qui s'adaptent à une $\mathrm{F}_{\mathrm{I}} \mathrm{O}_{2}$ plus basse en haute altitude manifestent: 1) une moins bonne vasoconstriction pulmonaire hypoxique; 2) un meilleur métabolisme glycolytique; 3) une densité microvasculaire et un volume sanguin accrus; et 4) une capacité accrue de transport de l'oxygène du sang. ${ }^{14} \mathrm{Ces}$ adaptations surviennent au niveau cellulaire et appuient le phénomène de préconditionnement hypoxique. ${ }^{15}$ Bien qu'il soit possible que nous ne comprenions jamais pleinement les mécanismes impliqués ici, on pourrait tester cliniquement l'hypothèse qu'un préconditionnement hypoxique léger et contrôlé pourrait améliorer les devenirs des patients après une anesthésie générale et une chirurgie. L'approche scientifique réductionniste n'ayant pas rencontré de succès clinique dans la recherche d'un mécanisme efficace de protection des organes, une approche généralisée de boîte noire pourrait être justifiée si l'on souhaite déterminer l'efficacité du préconditionnement hypoxique léger. Il faut cependant trouver le juste milieu en tenant compte des risques connus d'une hypoxie intermittente, notamment l'hypertension pulmonaire et la mortalité.

En conclusion, il faut féliciter Ehrenfeld et coll. ${ }^{1}$ pour leur observation d'une base de données clinique d'envergure et d'avoir mis en exergue que l'hypoxémie peropératoire demeure une préoccupation actuelle. Les pratiques qui réduisent le risque d'hypoxémie dans ce contexte, y compris la préoxygénation, une concentration peropératoire accrue de $\mathrm{F}_{\mathrm{I}} \mathrm{O}_{2}$ et la préservation d'une ventilation adaptée, sont essentielles à la prévention de l'hypoxémie peropératoire. Enfin, il faut chercher des approches innovantes destinées à minimiser la fréquence de l'hypoxémie liée à l'anesthésie, tout en continuant la recherche fondamentale et en science clinique afin de découvrir les mécanismes peut-être plus subtils qui y contribuent.

Acknowledgements We thank Drs. C.D. Mazer and V. Hughes for their insight and knowledge regarding the history of nitrous oxide induction.

Conflicts of interest None declared.

\section{References}

1. Ehrenfeld JM, Funk LM, Van Schalkwyk J, Merry A, Sandberg WS, Gawande A. The incidence of hypoxemia during surgery: evidence from two institutions. Can J Anesth 2010; 57: DOI: 10.1007/s12630-010-9366-5.

2. Mostert JW. Nitrous oxide anaesthesia without harm. Br Med J 1958; 1: 502-3. 
3. Clark LC Jr, Wolf R, Granger D, Taylor Z. Continuous recording of blood oxygen tensions by polarography. J Appl Physiol 1953; 6: 189-93.

4. Eger EI II. Nitrous Oxide / H2O. New York, Elsevier Science Publishing Company Inc., 1985.

5. Heller ML, Watson TR Jr. Arterial oxygenation during transition from 100 per cent oxygen to air breathing: polarographic $\mathrm{PaO} 2$ study. Anesthesiology 1961; 22: 385-92.

6. Schmitt EL, Baum VC. Nitrous oxide in pediatric anesthesia: friend or foe? Curr Opin Anaesthesiol 2008; 21: 356-9.

7. Sanders RD, Weimann J, Maze M. Biologic effects of nitrous oxide: a mechanistic and toxicologic review. Anesthesiology 2008; 109: 707-22.

8. Eger EI II, Severinghaus JW. Effect of uneven pulmonary distribution of blood and gas on induction with inhalation anesthetics. Anesthesiology 1964; 25: 620-6.

9. Gencorelli FJ, Fields RG, Litman RS. Complications during rapid sequence induction of general anesthesia in children: a benchmark study. Paediatr Anaesth 2010; 20: 421-4.
10. Constantin JM, Futier E, Cherprenet AL, et al. A recruitment maneuver increases oxygenation after intubation of hypoxemic intensive care unit patients: a randomized controlled study. Crit Care 2010; 14: R76.

11. Duggan $M$, Kavanagh BP. Pulmonary atelectasis: a pathogenic perioperative entity. Anesthesiology 2005; 102: 838-54.

12. Grasso F, Engelberts D, Helm E, et al. Negative-pressure ventilation: better oxygenation and less lung injury. Am J Respir Crit Care Med 2008; 177: 412-8.

13. Blum JM, Fetterman DM, Park PK, Morris M, Rosenberg AL. A description of intraoperative ventilator management and ventilation strategies in hypoxic patients. Anesth Analg 2010; 110: 1616-22.

14. Hochachka PW. Mechanism and evolution of hypoxia-tolerance in humans. J Exp Biol 1998; 201: 1243-54.

15. Zhang J, Qian H, Zhao P, Hong SS, Xia Y. Rapid hypoxia preconditioning protects cortical neurons from glutamate toxicity through delta-opioid receptor. Stroke 2006; 37: 1094-9. 